\title{
The Physiological Proof that Starling's Law for the Capillary-Interstitial Fluid Transfer is Wrong: Advancing the Porous Orifice (G) Tube Phenomenon as Replacement
}

\author{
Khaled A Ghanem ${ }^{1}$ and Ahmed N Ghanem ${ }^{2 *}$ \\ ${ }^{1}$ Mansoura University Hospital, Egypt \\ ${ }^{2}$ Retired Consultant Urologist, Egypt
}

*Corresponding author: Ahmed N Ghanem, Retired Consultant Urologist, No1 President Mubarak Street, Mansoura 35511, Egypt, Tel: 001020883243; Email:anmganem1@gmail.com

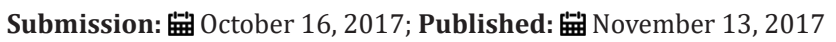

\begin{abstract}
Objective: To report physiological evidence that the capillary works as a porous orifice (G) tube proving that Starling's law is wrong.

Material and methods: The G tube has a negative side pressure (SP) exerted on its wall. The G tube is akin to capillary and when enclosed in a chamber (C), akin to interstitial fluid space, demonstrating the G-C circulation. Here we report the results of experiments on the hind limb of sheep; first when the fluid is passed through the artery and later when passed through the vein. We monitored the occurrence of oedema of the limb comparing the two circulations.
\end{abstract}

Results: The inflow pressure induces negative SP in the G tube which is responsible for absorption and G-C phenomenon: an autonomous magnetic field-like G-C circulation occurs between fluid in the G tube's lumen and a surrounding fluid compartment $\mathrm{C}$. The hind limb acted as normal without oedema and has a negative interstitial (ISF) pressure when the fluid is passed through the artery i.e. acting like the G tube. There was gross oedema with increase in weight of the limb when the fluid was passed through the vein acting like Poiseuille's tube.

Conclusion: The reported studies affirm that the capillary works as G tube effecting circulation with ISF which causes negative ISF pressure. When the circulation is reversed through the vein it acted like Poiseuille's tube causing gross oedema and positive ISF pressure. This is physiological proof that Starling's law is wrong.

Keywords: Capillary circulation; Starling's law; Capillary interstitial fluid transfer; Hydrodynamic; Shock; Hyponatraemia, Oedema the transurethral prostatectomy syndrome (TURS); the adult respiratory distress syndrome (ARDS)

Abbreviations: VO: Volumetric Overload; VOS: Volumetric Overload Shocks; VOS1: Volumetric Overload Shock, Type 1; VOS2: Volumetric Overload Shock, Type 2; TURP: The Transurethral Resection of the Prostate Syndrome; ARDS: The Adult Respiratory Distress Syndrome; MVOD/F: The Multiple Vital Organ Dysfunction/ Failure Syndrome; HN: Hyponatraemia; BP: Arterial Blood Pressure; CVP: Central Venous Pressure; ISF: Interstitial Fluid Volume; G Tube: The Porous Orifice Tube; PP: Proximal Pressure to the G tube akin to arterial blood pressure; DP: Distal Pressure to the G tube akin to venous pressure; LP: Lumen Pressure of the G tube; FP: Flow Pressure is the positive pressure inside the G Tube; SP: Side Pressure is the negative pressure inside the G Tube

\section{Key Points}

a) Dr. Starling assumed that the capillary works as Poiseuille's tube with positive side pressure on its wall causing filtration.

b) He also assumed that the capillary wall is impermeable to plasma proteins and oncotic pressure causes absorption.

c) Later it was discovered that the capillary has pre-capillary sphincter and is porous allowing passage of plasma proteins. d) The hydrodynamics of a porous orifice $(G)$ tube is different from Poiseuille's tube- it has a negative side pressure causing suction.

e) We did physiological experiments on hind limbs of sheep and found that capillaries work as G tube when fluid inflow through the artery causing absorption of fluid and negative interstitial tissue pressure. 
f) When inflow through the vein the capillary acted as Poiseuille's tube interstitial pressure becomes positive with oedema formation.

g) This is a physiological proof that Starling's law is wrong.

\section{Introduction}

In 1886, Starling [1] proposed a hypothesis for the capillaryinterstitial fluid (ISF) transfer in which the capillary was thought a tube of a uniform diameter that is impermeable to plasma proteins. The flow of fluid across its wall was thought dependent upon a balance between the hydrostatic pressure within its lumen, encouraging fluid to leave 'filtration', and the osmotic pressure of plasma proteins, tending to draw fluid back to the capillary lumen 'absorption', with similar opposing smaller forces in the ISF space. At the arterial end of the capillary, lumen pressure (LP) is greater than the oncotic pressure and fluid is pushed out. At the venous end, osmotic pressure is higher and fluid is withdrawn into capillary lumen.

The physical basis on which LP of a capillary was thought positive and responsible for filtration was Poiseuille's work (1799-1869) on long Brass tubes of uniform diameters [2]. However, Bernoulli's effect of a fluid jet and Venturi's effect of a tube constriction are well known and must also be considered to be of significance even under laminar flow conditions. LP refers to the arterial pressure of a capillary. Starling's hypothesis has undergone extensive evolution to become a law prior to discovery of the capillary ultra-structure of the pre-capillary sphincter [3] and its porous wall [4] plus the osmotic chemical composition [5], the negative ISF pressure [6], the dynamics of lymph flow [7]. Failure of Starling's law to explain the capillary-ISF transfer in many parts of the body [8], particularly vital organs, has previously called for reconsideration [9].

In 1984, clinical observations inconsistent with Starling's hypothesis prompted physical and clinical studies [10-12] to verify LP and fluid dynamics in a porous orifice $(G)$ tube with reference to the effect of the pre-capillary sphincter, arterial and venous pressures on the capillary-ISF transfer. The observation was: during hypotension shock in which prompt and adequate vascular expansion for resuscitation, all fluids leaked into and drowned the ISF space and filled the potential body cavities, demonstrated on post-mortem examination! Here we complete the work by providing physiological evidence that the capillary acts as G tube.

\section{The Questions and Logic}

The above observation, augmented with another that although arterial hypertension is very common it does not cause oedema, posed the questions: If LP is a filtering force in the capillary, how such massive fluid was filtered into ISF space during hypotension? Is LP truly responsible for filtration and, if not, what is? The logic that followed was that: if Dr Starling had based his hypothesis on Poiseuille's results of physical experiments, similar studies on tubes built on a scale to the capillary ultra-structure, i.e. the $G$ tube, should answer the questions. This has paved the way to provide the physiological evidence reported here.

\section{Perspective Review of Capillary Physiology}

Folkow et al. [2] stated that: 'The greatest discovery in medical science was made by William Harvey, who showed in 1628 that the heart pumped the blood around the circulatory system and understood that the circulation provided nourishment for the tissues'. Four centuries earlier, however, Ebn Al-Nafis (12201298), discovered the pulmonary circulation. Both the systemic and pulmonary circulations exist to provide for tissues' viability. Capillary circulation is directly responsible for this vital function in every organ and tissue. In 1886, Starling [1], a great physiologist, proposed a hypothesis for the capillary-ISF exchange. It was based on two known physical forces; LP based on Poiseuille's tube as a filtration force and the oncotic pressure as a re-absorption force. In 1929, Landis [13] measured LP of a capillary, by a cannula facing up stream, which was 32 and $12 \mathrm{mmHg}$ at the arterial and venous ends, respectively. This is akin to FP component of LP.

In 1948, Pappenheimer et al. [14] studied the quantitative changes in the weight of isolated hind limbs of dogs induced by alterations of the arterial and venous pressures. Oedema was induced by increasing the venous pressure in order to match any increase in the arterial pressure ( $\mathrm{mmHg}$ for $\mathrm{mmHg}$ ). Such, 'isogravimetric state', in which capillary filtration exceeds absorption, as would be expected with high venous pressure, was thought in support of Starling's hypothesis. The authors also found that the osmotic pressure of a concentrated plasma solution was 23-28mmHg in vitro. Ever since, most physiologists have accepted Starling's hypothesis as a physiological law.

However, most of the enlightening scientific evidence on microcirculation started to appear decades later. The ultrastructure of the pre-capillary sphincter [3] and its porous wall [4] were reported in 1967. In 1960, Mellander [15] showed that ISF 'absorption' increased after autonomic stimulation, which narrows the lumen of 'microvessels' and pre-capillary sphincter, and increases arterial pressure. In 1962, Hendry [5] measured the oncotic pressure of various body fluids and found it identical to that of the plasma, pointing out: 'the osmotic pressure of plasma proteins is too weak a force to return fluids back into the capillary lumen'. In 1963, Guyton et al. [6] measured ISF tissue pressure, using a sub-cutanously implanted perforated capsule, and found that it has a negative value of $-7 \mathrm{~cm}$ water.

In 1972, Calnan et al. [7] confirmed this finding and showed that molecules, including plasma proteins, pass freely and rapidly between the capillary blood and the implanted capsule and vice versa. In 1967, Rhodin [3] showed that the capillary tube is encircled by a cuff of smooth muscle fibres at its arteriolar junction, named the pre-capillary sphincter (3-5 Micro $\mathrm{m}$ ), which is the narrowest part of the whole vascular system. Also in 1967, Karnovesky [4] showed that the capillary wall is made of flat cells and their intercellular junctions are slits 10 to $20 \mathrm{~nm}$ wide, which are the pores through which fluids, nutrients and protein molecules pass freely. His photographs show the stained horse radish globules, which are much larger than plasma protein molecules, passing through these 
pores. The findings concerning plasma proteins and capillary pores alone, has cancelled the absorption force in Starling's hypothesis. After all, plasma proteins' main function, like blood glucose, must be a nutrient material for the cells.

In 1983, Mattfeldt et al. [16] reported the ultra-structure dimensions of capillaries. The 'ideal' capillary is a tube connecting an arteriole to a venule. According to Crogh's model it is a perfect, anisotropic, straight and un-branched tube with a diameter of 7-18 Micro $\mathrm{m}$. The pre-capillary sphincter and intercellular slits make the capillary a strait 'porous orifice tube', based on which the G tube was made on a larger scale.

In 1982, Keele et al. [8] as in Guyton [17], pointed out that the concentration of tissue proteins in the liver, lung and muscles is $60 \%$ that of plasma proteins. In the pulmonary circulation arterial pressure is less than the plasma oncotic pressure. Thus fluid filtration in the lungs and re-absorption in the liver and muscles lacks explanation. The oncotic pressure neither can explain the negative pressure in ISF space [6] nor the speed and efficiency with which the capillary-ISF fluid, nutrients and oxygen transfer occurs. Lymph drainage cannot explain the negative ISF pressure [6,7] either. This knowledge on capillary ultra-structure and permeability to macromolecules, prompted Renkin [9] to call for reconsideration of Starling's hypothesis but an alternative mechanism as reported here was still evolving at that time.

\section{Material and Methods}

We studied the hydrodynamics of a rubber inlet tube in order to demonstrate the negative side pressure (SP) gradient exerted on its wall as well as the flow pressure (FP) components of its lumen pressure (LP). We then studied the porous orifice (G) tube akin to capillary and later enclosed it in a chamber (C), akin to interstitial fluid space, making the G-C apparatus demonstrating the G-C circulation phenomenon. The results of physical experiments was reported [18,19] but summarized here in order to facilitate the understanding of the results of physiological experiments reported here. The factors affecting the speed and efficiency of the G-C circulation were evaluated. These included the proximal pressure (PP), the distal pressure (DP) and the inlet diameter (r) as a relation to the tube diameter (R). The G-C apparatus was enclosed in a circulatory model driven by electrical pump and connected to manometers for evaluating the hydrodynamics of the circulatory model.

Here we report the results of experiments on the isolated hind limbs of sheep. The hind limbs were bought from a butcher after freshly slaughtered and skinned. The hind limb was tightly wrapped in a Cling film to replace the skin. After finishing with the experiment the limb was nicely cooked and eaten. In every limb studied we ran fluid through the artery then later reversed the inflow to run through the vein observing the occurrence of oedema. We compared Normal Saline to Plasma Substitute (Haemaccel). Based on the results of physical experiments Poiseuille's tube has a positive SP on its wall causing fluid to come out. In the G tube the SP on its wall is negative gradient causing fluid to be sucked in over the proximal part and come out over the distal part. Thus if the capillary works as Poiseuille's tube it should make no difference from which end the fluid flows through it: the fluid will filter and cause oedema in both cases. But if the capillary works as G tube during arterial inflow it will demonstrate negative SP on the capillary wall that reflects on the ISF pressure without oedema formation.

\section{Results}

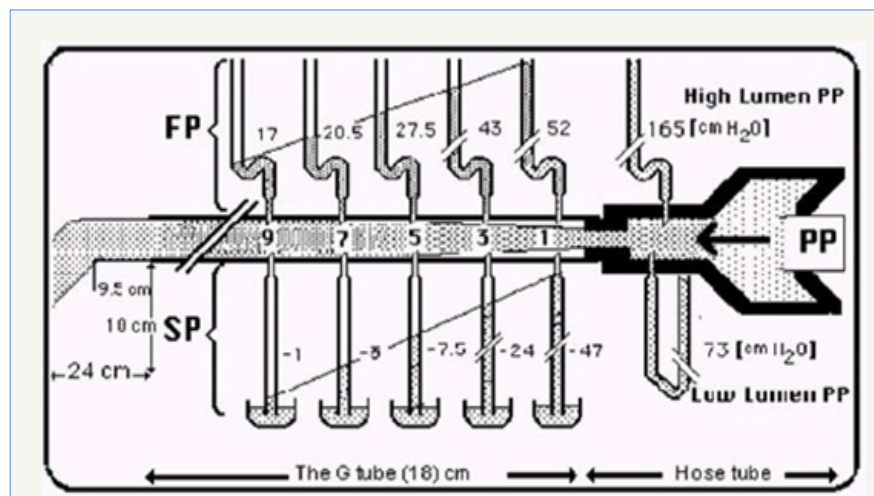

Figure 1: Shows the lumen pressure (LP) components of flow pressure (FP) and side pressure (SP) of a rubber orifice tube as measured by manometers with needles inserted at various $\mathrm{cm}$ distances from the inlet. When the needle's bevel faces upstream it measures FP (Top manometers) and when facing downstream it measures SP (Bottom manometers).

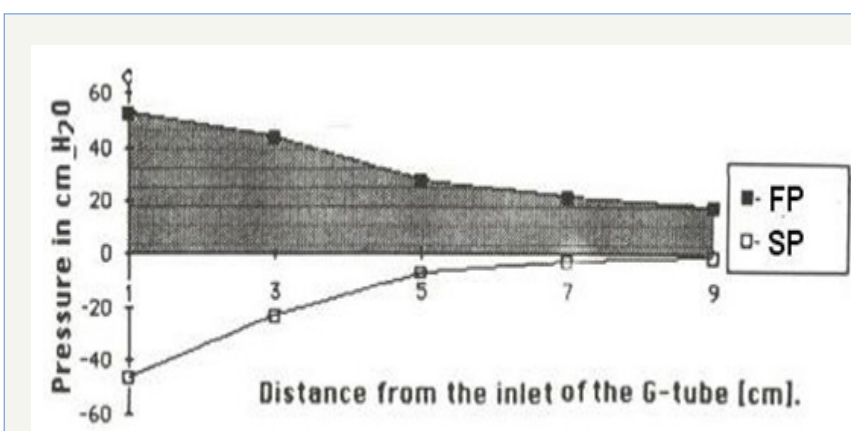

Figure 2: Graph showing the FP and SP components of LP of the $\mathrm{G}$ Tube at $\mathrm{cm}$ distances from the inlet.

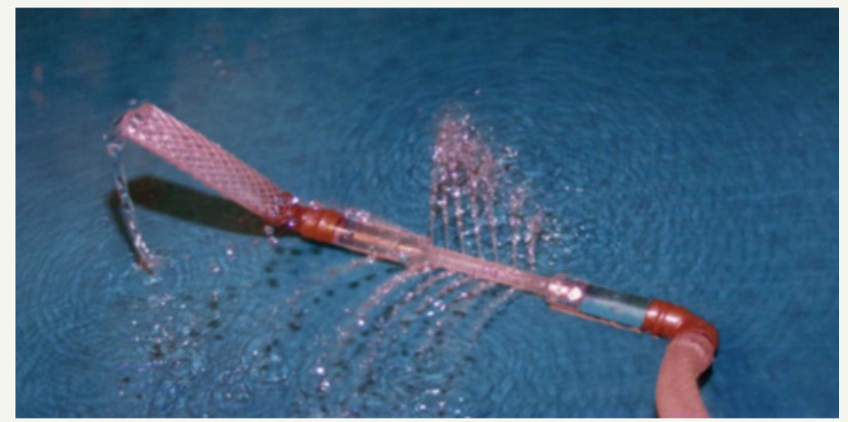

Figure 3: Shows the hydrodynamic of a porous orifice (G) tube. The side pressure (SP) gradient exerted on its wall turns from negative near the inlet to positive near the exit. The magnetic field like G-C circulation is shown when the tube is placed in a surrounding chamber but can be seen at the top of the photo. 
The hydrodynamics of a rubber inlet tube demonstrates the negative side pressure (SP) gradient exerted on its wall as well as the flow pressure (FP) components of its lumen pressure (LP) is shown in Figure 1. A graph showing FP and SP gradients is shown in Figure 2. The hydrodynamics of the $\mathrm{G}$ tube is shown in Figure 3. The G-C phenomenon is shown in Figure 4. The relation of PP to SP and $\mathrm{CP}$ is shown in Figure 5. The relation of orifice diameter to SP and CP is inverted Bell shaped, and is shown in Figure $6 \& 7$. The relation of DP to SP and CP is shown in Figure 8. The pressure gradient measured at various points in the G- C circulatory model is shown in Figure 9. Figure 10 shows a circulatory model incorporating the G-C apparatus with manometers measuring various pressures. These figures were previously reported [18]. In the physiological experiments we replaced the G-C model with the isolated hind limb.

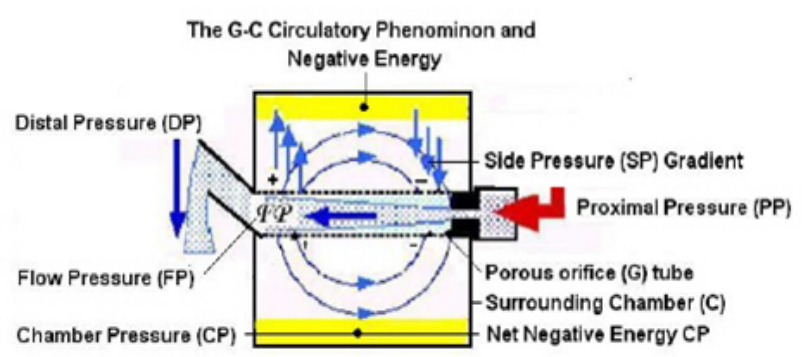

Figure 4: Shows a diagram of the G-C circulation based on several photographs creating a net negative pressure in $\mathrm{C}$ (Highlighted in yellow).

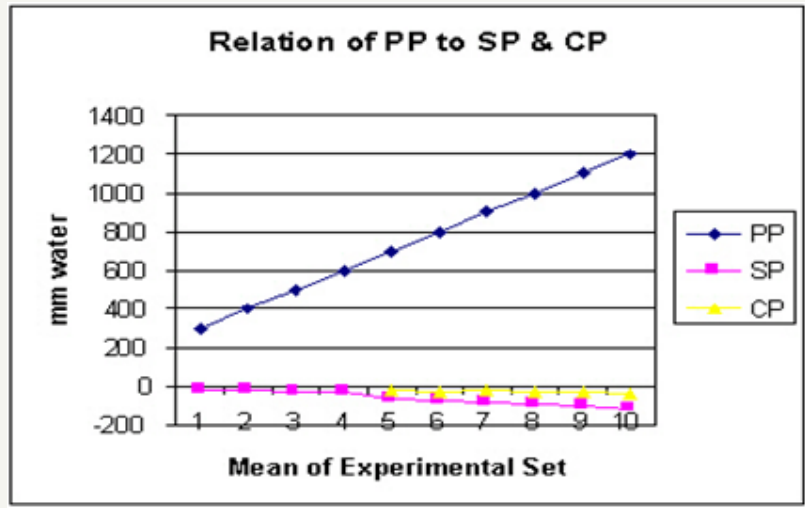

Figure 5: Shows the relation of proximal pressure (PP) to side pressure (SP) and chamber pressure (CP). A high PP enhances suction negativity of SP and $\mathrm{CP}$

It is observed from the presented results that the hydrodynamics of the G tube is totally different from Poiseuille's tube. The orifice of the $\mathrm{G}$ tube creates a negative pressure gradient on its wall inducing a suction force which is transmitted to the surrounding chamber C creating a dynamic magnetic field like fluid G-C circulation that rapidly irrigates the $C$. The orifice thus transfers the PP from a filtration force in Poiseuille's tube into a suction force in the $\mathrm{G}$ tube. Increasing the $\mathrm{PP}$ enhances the $\mathrm{G}-\mathrm{C}$ circulation while reducing it slows it down as shown in Figure 5. The distal (venous) pressure has the opposite effect. Increasing the DP slows down the G-C circulation and turns the pressure in $\mathrm{C}$ into positive with increased volume. The effect of increasing the orifice diameter has an inverted Bell shaped effect on SP and CP Figure 6. The G-C circulation thus offers a complete and correct replacement for Starling's law. The circulatory model Figure 10 has remarkable similarity to the circulatory vascular system.

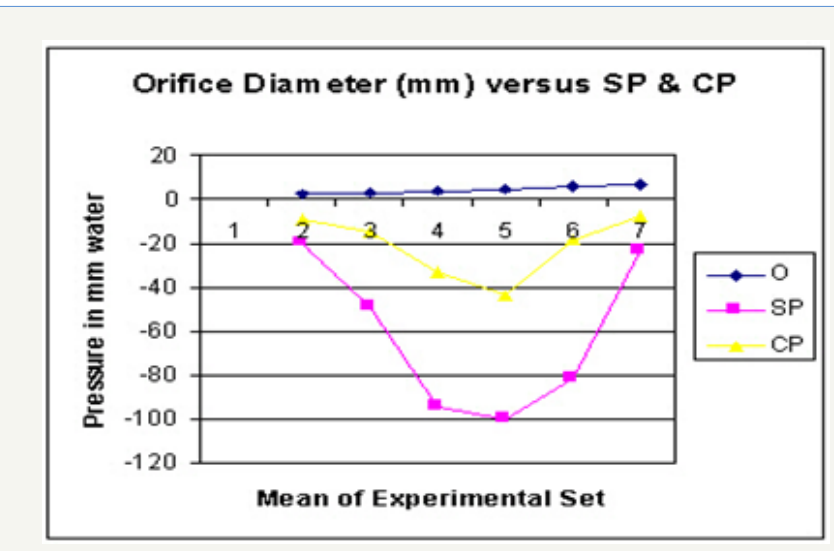

Figure 6: Shows the relation of orifice diameter $(\mathrm{O})$ to side pressure (SP) and chamber pressure (CP).

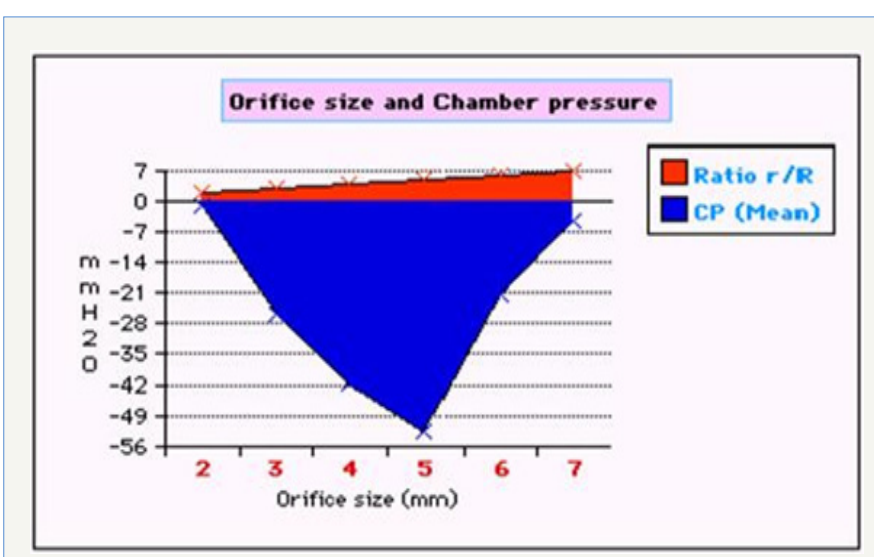

Figure 7: Shows the relation of orifice ratio to tube diameter and effect on $\mathrm{CP}$.

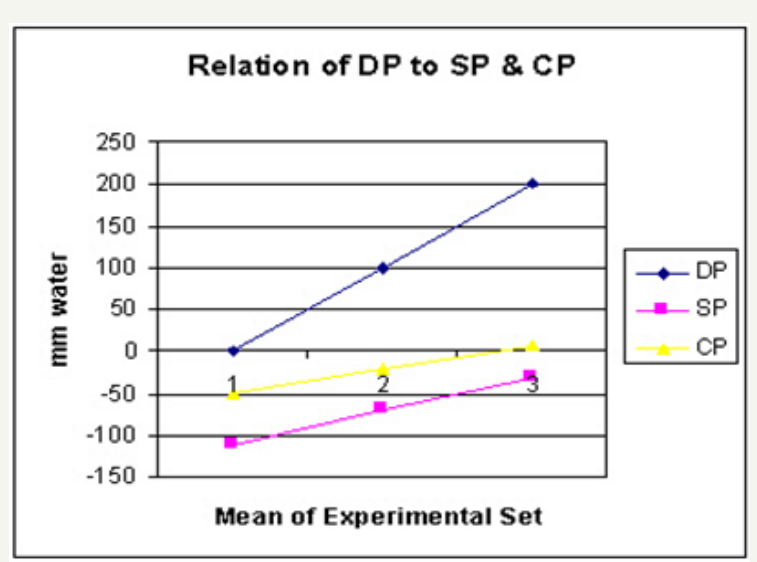

Figure 8: Shows the relation of distal (venous) pressure (DP) to side pressure (SP) and chamber pressure (CP). 


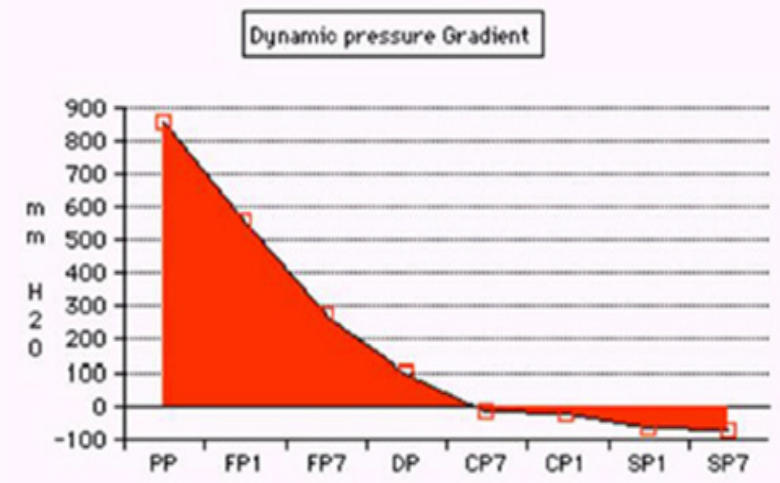

Figure 9: Shows the pressure gradient from proximal pressure (PP) to flow pressure (FP) inside the G tube at points 1 and 7 to distal venous pressure (DP) then chamber pressure (CP) and side pressure (SP) at points 1 and 7 .

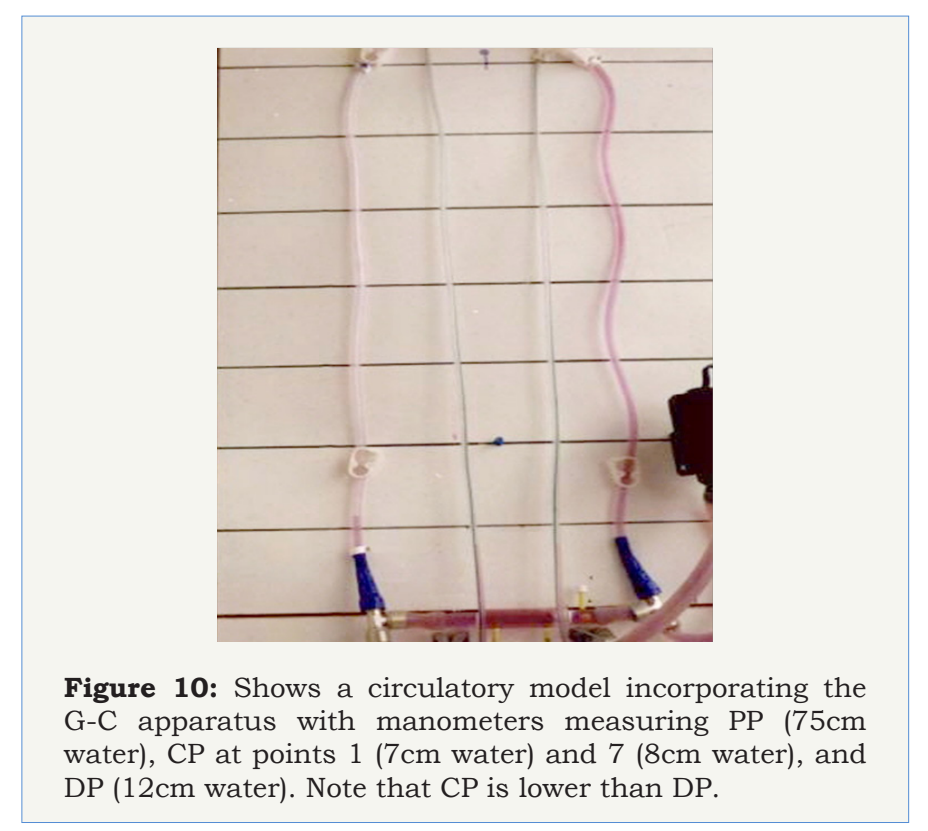

In the physiological experiments running the fluid through the artery made the capillary act like a G tube causing suction through its proximal part and filtration through the distal part inducing a rapid circulation between the capillary lumen and surrounding ISF space with net negative pressure in the ISF space i.e. without oedema formation. Reversing the circulation and making fluid to run through the vein caused positive SP with filtration causing massive oedema with increased weight of the limb. Changing the circulating fluid from Normal Saline to Plasma Substitute (Haeemaccel) made no difference on the limb during both arterial and venous circulations. The absence of oncotic pressure on using Normal Saline does not cause oedema. Reversing the inflow from venous back to arterial caused absorption of all the accumulated fluid in the ISF space and restored its negative pressure. This is a direct physiological proof that Starling's Law is wrong and the hydrodynamics of the $\mathrm{G}$ tube provide the correct alternative.

\section{Discussion}

Results of studies on the G tubes would make sense when linked to its physiological and haemodynamic equivalents. PP akin to 'arterial' pressure induces a flow jet with its LP components of FP and SP. The negative energy SP component occurs maximally over the proximal part of the G tube (Figure 1), and is mainly responsible for suction akin to 'capillary absorption'. Filtration occurs autonomously over the distal part of the G tube (Figure $1 \& 2$ ). Both effects induce the G-C circulation phenomenon (Figure 3). SP also induces a net negative energy pressure in chamber C (Figure 4), demonstrated by the caving in of a membrane cover. A net negative $\mathrm{CP}$ is akin to the negative pressure of ISF and subcutaneous spaces $[6,7]$. Although fluid comes out autonomously through the distal holes of the $\mathrm{G}$ tube, it is greatly augmented by increasing the outflow DP akin to elevating venous pressure, augmenting 'filtration' and causing ISF shift or oedema.

The G-C circulation phenomenon causes rapid mixing of fluids in the lumen of the $\mathrm{G}$ tube and a surrounding fluid chamber compartment C (Figure 4). Its efficiency in exchanging materials between the $\mathrm{G}$ tube and chamber $\mathrm{C}$, akin to 'capillary and ISF compartments' is remarkable. A few small $G$ tubes contained in chamber $\mathrm{C}$ have commutative effect. The shape of pores, whether hole or slit makes no difference. Large particles trapped in chamber $\mathrm{C}$ require regular cleaning. The negative energy of the $\mathrm{G}-\mathrm{C}$ circulation is a considerable force. This negative energy force effectively irrigates chamber $\mathrm{C}$, allowing rapid transfer of fluid and particles into and from chamber $\mathrm{C}$, akin to the passage of fluids with 'oxygen and nutrients including protein molecules into the ISF space and washing out waste products while preventing excess ISF accumulation. Guyton and Coleman's capsule [6] provided excellent evidence on the negative energy pressure of the ISF induced by the dynamic capillary flow, as demonstrated by the net negative CP in the G-C apparatus (Figure 4). The presented evidence demonstrates that the capillaries act like $G$ tubes in every aspect of the experiments. It allows rapid capillary ISF transfer inducing a net negative pressure in the ISF space which prevents fluid accumulation in ISF space and oedema formation. On the other hand making capillary act as Poiseuille's tube by reversing the flow through the vein caused massive oedema formation and positive ISF pressure with matching increase in the limb's weight.

Analysis of the forces which initiate and regulate the G-C circulation with reference to its haemodynamic equivalents is best demonstrated by comparing the dynamics of PP, DP and CP of the G-C apparatus incorporated in a circulatory model (Figure 10). Before starting the pump an adequate volume of fluid induces a hydrostatic equilibrium in all manometer tubes. Running the pump induces the dynamic pressure values in the manometers. In (Figure 10), $P P=650$ and $D P=12$ while $C P$ had values of 8 and $10 \mathrm{~mm}$ waters at points 1 and 2 in chamber C. Fluid in chamber $C$ flows from point 2 to point 1 , in an opposite direction to flow in the G-C circulatory system model, shown by injecting ink into chamber C. Adding more fluid to the system mimicking VO or vascular expansion increases venous pressure and $\mathrm{CP}$. 
This means that increasing DP elevates CP and increases fluid volume in chamber $\mathrm{C}$ by a proportional amount of hydrostatic pressure. A pressure gradient remains between DP, CP2 and CP1, allowing fluid movement down it. Massive VO of the system model increased fluid volume and $\mathrm{CP}$ in chamber $\mathrm{C}$, which decreased the negative energy SP and slowed down significantly the G-C circulation. Most interestingly, such VO also reduced PP. The injected ink into chamber $\mathrm{C}$ moves very slowly in the opposite direction to fluid flow in the G-C circulatory system. Volumetric expansion until DP, CP and PP attain hydrostatic equilibrium ceases the G-C circulation even while the pump is operating. This may mimic a pathological haemodynamic circulatory condition.

Received thinking that elevating CVP is synonymous with elevating arterial pressure is prevailing in current clinical practice during fluid therapy for shock and the management of the acutely ill patients. This is undoubtedly correct during restoration therapy for hypovolaemic and haemorrhagic shock. But, vascular expansion or VO is a different issue. Persistent attempts to elevate CVP and PCWP up to levels of $15 / 18 \mathrm{mmHg}$ are commonly received but mistaken practice. The normal CVP is around 0 and most textbooks report a range of -7 to $+7 \mathrm{~cm}$ water [17].

Vascular expansion causes volumetric overload shocks (VOS) [18-22]. The pathological effects of vascular VOS have been overlooked for various reasons [18]. Perhaps, because the above reported sophisticated components of a dynamic flow were unknown. Hypotension is always thought synonymous with hypovolaemia. Yet, vascular expansion may not elevate arterial pressure and at times, it aggravates hypotension [18-22]. The maximal capacitance of an adult's cardiovascular system is about 7 liters [17] and any excess VO overflows into the ISF space, causing flooding and drowning. Thus, both hypovolaemia and hypervolaemia of 2 liters have pathological haemodynamic effects. Understanding the correct mechanism of the capillary-ISF transfer in relation to CVS volume, capacitance and dynamic pressures in regulating a physiological capillary-ISF circulation is clinically important. It is relevant to the management of ISF shift, oedema, shock and the MVOD/F syndrome [18-22].

The autonomous magnetic field-like G-C circulation is the concept proposed as a mechanism for a new capillary-ISF hypothesis. The reported physiological evidence directly supports the physical evidence on the $G$ tube. The speed and autonomy of the G-C circulation, under similar ranges of dynamic pressures of a human circulatory system, can truly explain the efficiency of capillary- ISF circulation in transporting oxygen and nutrients into cells while removing carbon dioxide and metabolites from ISF space. It should work in every part and organ of the body under both physiological and pathological conditions.

Apart from suggesting that fluid flows in at the arterial end and out at the venous end, through the wide slit pores of the capillary wall, the reported results also suggest that both the absorption and filtration are autonomous. Venous pressure rather than arterial pressure, affects filtration while re-absorption is the primary effect of arterial pressure and orifice or 'pre-capillary sphincter'. This explains the observation that arterial hypertension, though common, never causes oedema while an increase in venous pressure does. It is in agreement with the results of physiological studies. More importantly, it may resolve the puzzle of the MVOD/F syndrome known as the adult respiratory distress syndrome [1822].

The high incidence of the MVOD/F syndrome affecting postsurgical and trauma patients with its high morbidity and mortality [18-22] is a subject of increasing international attention, concern and anxiety. Although, the role of microcirculation in its pathophysiology is recognized, it's a etiology has remained elusive and the possible role of haemodynamic pressures and VO in its pathogenesis has been overlooked but has been recently reported [18-22]. VO shocks in which vascular expansion, aimed at correcting arterial hypotension by elevating CVP and PCWP to levels up to 15 and $18 \mathrm{mmHg}$, may culminate into the MVOD/F syndrome [11,1822] better known as the adult respiratory distress syndrome. Massive fluid shift drowns the ISF and/or intracellular spaces, depending on fluid type.

The transurethral resection of the prostate (TURP) syndrome is induced by massive sodium-free fluid overload (V01), induced mostly by irrigant absorption during endoscopic surgery. The diluted serum solutes are good serum markers, characterized by dilution hyponatraemic encephalopathic coma among features of the MVOD/F syndrome. The main haemodynamic disturbance of V01 is hypotensive shock with interstitial fluid shift initially and intracellular fluid shift later [11,18-22]. Despite the massive V0, the patient suffers from severe irreversible hypotension shock and appears hypovolaemic. Ironically and misleadingly, this shock induced by massive V01 calls for further vascular expansion! Sodium-based fluids (VO2) are commonly used to combat this shock. It transfers VOS1 into VOS2, erases serum markers and causes internal drowning while establishing the MVOD/F syndrome [18-22]. Such situation may also complicate the management of any type of the recognized shocks using any type of VO2 fluids.

\section{Conclusion}

Hydrodynamic studies on a porous orifice $(G)$ tube, based on capillary ultra-structure, demonstrate results which differ from Poiseuille's in a strait tube and hence challenge the role attributed to arterial pressure as a filtration force in Starling's hypothesis. A perspective literature review shows that the oncotic pressure force has been previously cancelled and the hypothesis has failed to explain the capillary-ISF transfer in most parts of the body.

A concept based on a new hydrodynamic phenomenon of the $\mathrm{G}$ tube is proposed for the capillary-ISF circulation hypothesis. It explains this vital circulation in every organ and tissue under both physiological and pathological conditions. An autonomous dynamic magnetic field-like G-C circulation occurs between fluid in the $G$ tube's lumen or capillary and a surrounding fluid compartment $\mathrm{C}$ or ISF space. Based on results of studies on a circulatory model 
incorporating the G-C apparatus, the capillary- ISF transfer is reported in the hind limbs as physiological evidence reported here. Factors which initiate, regulate and affect the G-C circulation are reported. The physiological and haemodynamic relevance and its clinical importance to the pathogenesis of oedema, shock and the MVOD/F syndrome are discussed.

\section{References}

1. Starling EH (1886) Factors involved in the causation of dropsy. Lancet 1266-1410.

2. Folkow B, Neil E (1971) Circulation. Oxford University Press, England, UK, pp. 1-125.

3. Rhodin JA (1967) The ultra structure of mammalian arterioles and precapillary sphincters. J Ultrastructure Research 18(2): 181-222.

4. Karnovesky MJ (1967) The ultra structural basis of capillary permeability studied with peroxidase as a tracer. J Cell Biol 35(1): 223-236.

5. Hendry EB (1962) The osmotic pressure and chemical composition of human body fluids. Clin Chem 8(3): 246-265.

6. Guyton AC, Coleman TG (1968) Regulation of interstitial fluid volume and pressure. Ann N Y Acad Sci 150(3): 537-547.

7. Calnan JS, Pflug JJ, Chisholm GD, Taylor LM (1972) Lymphatic surgery. Proceedings Royal Soc Med 65: 715-719.

8. Keele CA, Neil E, Joels N (1982) Sampson Wright Applied Physiology $\left(13^{\text {th }}\right.$ edn), Oxford University Press, England, UK.

9. Renkin EM (1986) Some consequences of capillary permeability to macromolecules: Starling's hypothesis reconsidered. Am J Physiol 250(5): H706-H710.

10. Ghanem AN (2001) Magnetic field-like fluid circulation of a porous orifice tube and its relevance to the capillary interstitial fluid circulation: preliminary report. Med Hypotheses 56(3): 325-334.

11. Ghanem AN (1988) The Transurethral Prostatectomy (TURP) Syndrome: An Investigation of the Osmotic and Metabolic Sequelae of Volumetric Overload. MD Thesis. Institute of Urology and Nephrology, Mansoura University, Mansoura, Egypt.
12. Ghanem AN, Ward JP (1990) Osmotic and metabolic sequelae of volumetric overload in relation to the TURP syndrome. Br J Urol 66(1): $71-78$

13. Landis EM (1929) Micro-injection studies of capillary blood pressure in human skin. Heart 31(15): 209-228.

14. Pappenheimer JR, Rivera S (1948) Effective osmotic pressure of plasma proteins and other quantities associated with capillary circulation in the hind limbs of cats and dogs. Am J Physiol 152(3): 471-491.

15. Mellander S (1960) Comparative studies on the adrenergic neurohormonal control of resistance and capacitance blood vessels in the cat. Acta Physiol Scand 176: 1-86.

16. Mattfeldt T, Mall G (1983) Estimation of length and surface of anisotropic capillaries. J Microsc 135: 181-190.

17. Guyton AC (1986) Textbook of Medical Physiology. An HBJ International ( $7^{\text {th }}$ edn), WB Saunders Company, Philadelphia London, UK.

18 Ghanem KA, Ghanem AN (2017) The proof and reasons that Starling's law for the capillary-interstitial fluid transfer is wrong, advancing the hydrodynamics of a porous orifice $(G)$ tube as the real mechanism. Blood Heart and Circ 1(1): 1-7.

19 Ghanem AN, Ghanem SA (2016) Volumetric overload shocks: why is starling's law for capillary interstitial fluid transfer wrong? the hydrodynamics of a porous orifice tube as alternative. Surgical Science 7(6): 245-249.

20 Nisha P, Salma GA, Khalid GA, Ahmed GN (2017) Volumetric overload shocks in the pathoetiology of the transurethral resection prostatectomy syndrome and acute dilution hyponatraemia. Integr Mol Med 4(2): 1-5.

21 Ghanem Khaled A, Ghanem Ahmed N (2017) Volumetric overload shocks in the patho-etiology of the transurethral resection prostatectomy syndrome and acute dilution hyponatraemia: The clinical evidence based on 23 case series. Basic Research Journal of Medicine and Clinical Sciences.

22 Ghanem SA, Ghanem KA, Ghanem AN (2017) Volumetric Overload Shocks in the Patho-Etiology of the Transurethral Resection of the Prostate (TURP) syndrome and acute dilution hyponatraemia: the clinical evidence based on prospective clinical study of 100 consecutive turp patients. Surg Med Open Access J 1(1): SMOAJ.000501. 